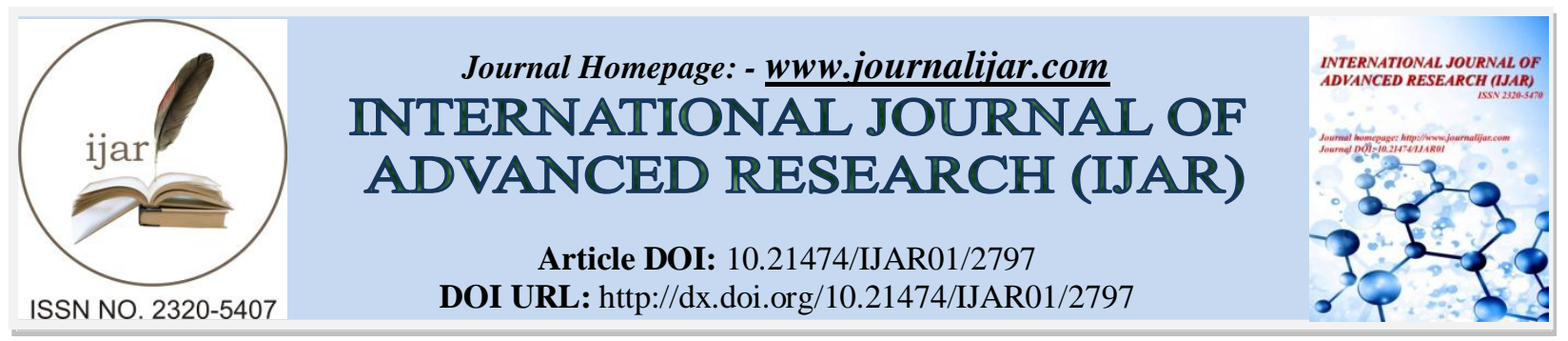

RESEARCH ARTICLE

\title{
EVALUATION OF SYSTOLIC DYSFUNCTION IN PATIENTS WITH LYMPHOPROLIFERATIVE DISORDERS AFTER STEM CELL TRANSPLANT: A SINGLE CENTER EGYPTIAN EXPERIENCE.
}

\author{
Haitham Mohammed Abdelbary, Yasmin Ahmed Abou El Magd, Gihan Kamal Shams Eldin and Mohamed \\ Osman Azzazi.
}

Clinical Hematology and Bone Marrow Transplantation Unit, Division of Internal Medicine, Ain Shams University, Cairo, Egypt.

\section{Manuscript Info}

Manuscript History

Received: 15 November 2016

Final Accepted: 17 December 2016

Published: January 2017

\section{Abstract}

Background: The long-term prognosis after Hematopoietic stem cell transplantation (HSCT) has greatly improved. However, HSCT survivors are at risk of developing long term complications. Cardiac complications are potentially life-threatening and there is a need to elucidate the incidence, severity and underlying mechanisms of these complications. We assessed the systolic cardiac function by echocardiography in patients who underwent autologus and allogeneic hematopoietic stem cell transplantation for lymphoproliferative disorders and evaluated the association between the conditioning regimens used and the decline in ejection fraction.

Materials and methods: 100 patients were enrolled in this retrospective study, 72 patients underwent autologus and 21 patients underwent allogeneic stem cell transplantation. 39 patients had multiple myeloma, 30 patients had NHL, 21 patients had acute lymphoblastic lymphoma, 10 patients had HD. LVEF was assessed by echocardiography and compared to a healthy control group as well as 50 positive control of patients who received chemotherapy without undergoing transplant.

Results: The mean ejection fraction of cases after transplant is significantly reduced compared to the negative control group, The ejection fraction decline was highly pronounced in older age groups, male patients and more in patients with larger surface area, with the use of TBI-based regimens followed by patients who received highdose cyclophosphamide in the conditioning regimens.

Conclusion: LVEF was reduced in patients with lymphoproliferative disorders after autologus and allogeneic hematopoietic stem cell transplantation and was correlated with the use of TBI-based conditioning regimens and high-dose cyclophosphamide.

Copy Right, IJAR, 2016,. All rights reserved.

\section{Introduction:-}

Allogeneic or autologous HSCT offers a possibility of cure or long-term remission in hematological malignancies. However, gastrointestinal, pulmonary, infectious and immunological complications are major problems and limiting factors for $\mathrm{HSCT}^{1}$. 
Finally, in the last few years reduced-intensity conditioning regimens have been applied to patients undergoing allogeneic HSCT to reduce drug toxicity while maintaining the potential for achieving disease remission exploiting the graft-versus-leukemia effect ${ }^{2}$.

The long-term prognosis after haematopoietic stem cell transplantation (HSCT) has greatly improved. However, HSCT survivors are at risk of developing long term complications, and mortality rates remain four- to ninefold higher than in the general population ${ }^{3}$.

Cardiac complications have been documented in several series, with reported incidence varying among investigators from 0 to $43 \%$, and mortality up to $9 \%$ in earlier studies ${ }^{1}$.

Cardiovascular complications can be grouped into three main categories: vascular abnormalities, cardiac structural problems and myocardial dysfunction and heart failure.

Evaluation of cardiac toxicity in patients includes consideration of biomarkers such as cardiac troponins and B-type natriuretic peptides, together with noninvasive imaging in the form of 2D-, 3D-, or strain echocardiography, multiple gated radionuclide angiography, quantitative gated blood-pool SPECT, or cardiac magnetic resonance imaging ${ }^{3}$.

The purpose of this study is to evaluate systolic dysfunction in patients with lymphoproliferative disorders after hematopoietic stem cell transplantation.

\section{Patients and Methods:- \\ Study Design:- \\ Patients and Control:-}

The study was conducted in the bone marrow transplantation unit (department of clinical hematology at Ain Shams university hospital) during a period from June 2015 to August 2016.

\section{Participants were grouped into:-}

Group (1): included 100 patients with lymphoproliferative disorders who underwent BMT in our unit, 79 patients underwent autologus transplant and 21 underwent allogeneic transplant, 30 patients with NHL, 10 patients with HD, 39 patients with multiple myeloma and 21 patients with acute lymphoblastic leukemia.

Group (2) (healthy control group): included participants without known cardiovascular disease, hypertension or DM. They were matched 1:1 based on age, sex, blood pressure and surface area with the cases.

Group (3) (positive control): 50 patients with lymphoproliferative disorders who received chemotherapy without undergoing BMT. They included 25 males and 25 females, $46 \%$ had MM, 42\% had NHL and $12 \%$ had HD.

Informed consents were obtained from all participants. The study was conducted in accordance with the stipulations of the local ethical and scientific committees of Ain Shams University and the procedures respected the ethical standards in Helsinki declaration of 1964.

\section{Methods:-}

All patients were subjected to full history and physical examination including the presence of B-symptoms like weight loss, fever, drenching sweats and pruritis, anemic manifestations or bleeding or the presence of bone fractures and the presence of lymphadenopathies or hepatosplenomegaly.

\section{To confirm the diagnosis of NHL and HD:-}

- Excision lymph node biopsy for fresh frozen and formaline-fixed samples for histopathological assessment.

- Immunohistochemistry for B-cell markers like CD20,CD19,CD79a,CD10 and T-cell markers like CD3,CD4,CD8,CD7,CD5,CD2, markers of immature lymphoblast like TdT, immunoglobulin Kappa and Lambda light chain restriction and for CD15 and CD30 for characterization of Reed-Sternberg cells.

- Chest X-ray, contrast-enhanced CT scans of the neck, chest and pelviabdomen.

- Baseline, interim and end of therapy PET was carried out whenever available for staging and response assessment. 
- Bone marrow aspiration and trephine biopsy.

- Cytogenetic analysis using conventional cytogenetics and FISH for certain specific chromosomal abnormalities.

- Staging is carried out according to Ann-Arbor staging system Examination.

- Cerebrospinal fluid and triple intrathecal chemotherapy prophylaxis was given to certain patients like: (1): patients with DLBCL with bone marrow, testicular, paranasal and epidural involvement. (2): lymphoblastic lymphoma. (3): primary CNS lymphoma.

- Upper GI endoscopy in those with suspected GI involvement eg. Mantle cell lymphoma.

To confirm the diagnosis of multiple myeloma:-

- Complete blood counts and serum chemistries especially for serum calcium, serum creatinine to detect CRAB features.

- Detection of monoclonal M-protein in the serum and urine by serum protein electrophoresis.

- Characterization of heavy and light chains in the serum and urine sample by immunofixation.

- Bone marrow aspiration and biopsy to evaluate the percentage of plasma cells.

- Conventional chromosomal analysis and FISH for high-risk myeloma like t(4;14), (14;16) and 17p.

- Skeletal survey for evaluation of lytic bone lesions using X-ray of spine, skull, pelvis, humerus and femur.

- MRI to evaluate symptomatic bony sites even if skeletal survey is negative or in case of spinal cord compression.

- Serum free light chains and Kappa/Lambda ratio for the detection of stringent CR whenever possible.

To confirm the diagnosis of acute lymphoblastic leukemia:-

- Complete blood count and differential for the detection of peripheral blasts.

- Bone marrow aspiration and trephine biopsy for the detection of the percentage of blast cells.

- Immunophenotyping for lymphoid and myeloid markers.

- Conventional cytogenetics and FISH for Philadelphia chromosome.

- $\quad$ T T scans for the neck, chest and pelviabdomen.

- CSF examination and triple intrathecal chemotherapy for prophylaxis and/or therapy in case of CNS involvement.

Patients who underwent autologus transplant were subjected to:-

$>$ Complete blood count using (LH Beckman coulter).

$>$ Serum chemistries including liver and renal function tests (AU 680 chemistry autoanalayzer).

$>$ Viral markers for hepatitis $\mathrm{B}$, hepatitis $\mathrm{C}$ and HIV using Rosh diagnostics and performed by copus instruments.

$>$ Polymerse chain reaction (PCR) for CMV using kits supplied by Qiagen and performed by rotor gene instrument for automated real time PCR.

> Serology for herpes simplex (HSV), Toxoplasma and Ebstein Bar virus (EBV).

$>$ Pulmonary function tests.

$>$ Renal scan.

$>$ Electrocardiogram and echocardiography.

$>$ Mobilization was done using different regimens ranging from G-CSF alone to G-CSF and cyclophosphamide or other chemotherapeutic regimen depending on the specific disease.

$>$ Conditioning regimens: for patients with MM high dose melphalan $200 \mathrm{mg} / \mathrm{m}^{2}$ on day -2 , for patients with NHL and $\mathrm{HD}$ they received a conditioning regimens consisted of cyclophosphamide $60 \mathrm{mg} / \mathrm{kg} / \mathrm{d}-3$ and -2 , etoposide $15 \mathrm{mg} / \mathrm{kg} / \mathrm{d}-3$ and -2 , carboplatin $400 \mathrm{mg} / \mathrm{m}^{2}-3$ and -2 .

In addition, patients undergoing allogeneic transplant are subjected to:-

- Donors received (after informed consent) 4 days of treatment with SC G-CSF (10ug/kg/d) before stem cells were collected.

- Mononuclear cells were isolated using a Cobe Spectra separator (Lakewood, Co, USA).

- The number of CD34 cells transfused was calculated using flowcytometric analysis.

- GVHD prophylaxis consisted of cyclosporine and methotrexate.

- Engraftment was defined as absolute neutrophilic count of more than 500 for three consecutive days.

- Chimerism analysis by variable number of tandem repeats (VNTR) was done at +28 and +56 after transplant. 
- Conditioning regimen consisted of TBI $2.5 \mathrm{GY}$ days -7 to -4 /Cyclophosphamide $60 \mathrm{mg} / \mathrm{kg}$ days -3 and -2 , TBI $2.5 \mathrm{GY}$ days -7 to -4 /etoposide $60 \mathrm{mg} / \mathrm{kg} \mathrm{d}-3$, Fludarabine $30 \mathrm{mg} / \mathrm{m}^{2}$ days -6 to -2 /oral Busulfan $1 \mathrm{mg} / \mathrm{kg} / 6$ hours days -6 to -3 .

All patients in both autologus and allogeneic transplant were treated using the same anti-infectious and transfusion policy of our transplant center.

Transthoracic echocardiography:-

- All patients were evaluated during transplant in case of occurrence of any minor or major cardiac events according to the international guidelines recommended by ACC and AHA.

- Transthoracic echocardiograph was done before transplant and 6 months after transplant.

- Transthoracic echocardiography was carried out using 2-4 MHz phased array transducer attached to a vivid S5 echocardiography machine by a cardiologist who was blinded to clinical details of each subject. Inter-observer variability was reduced by taking the mean of three reading during each echocardiography.

- The following parameters were examined: left ventricular end-diastolic diameter (LVEDD) and left ventricular end-systolic diameter (LVESD), intraventricular end-diastolic diameter (IVSDD), posterior wall end-diastolic diameter (PWEDD), right ventricular end-diastolic diameter (RVEDD), left atrium diameter (LA) and aorta diameter (Ao). The systolic function was determined by left ventricular ejection fraction (LVEF) using M-mode and modified Simpson's formula. Left ventricular diastolic function was evaluated with pulsed Doppler and tissue Doppler imaging (TDI). The following parameters of the diastolic function of left ventricle were determined: early filling velocity $\mathrm{E}$ wave (E) and A wave (A) of mitral inflow, Doppler-derived mitral deceleration time of early filling (DT), isovolumetric relaxation time (IVRT) and early diastolic velocity of mitral annulus wave (E0).

\section{Statistical analysis:-}

$>$ All analyses of the present study were done using SPSS version 17 software.

$>$ Statistical presentation and analysis of the present study was conducted, using the mean, standard deviation, student t-test, chi square.

$>$ Data were expressed as mean value \pm SD for continuous variables, and as percentages for categoric variables. In this study, statistical significance was established as follows: $p>0.05$ insignificant, $p \leq 0.05$ significant, $p \leq 0.01$ highly significant.

$>$ Comparisons between continuous variables were performed using the paired t-test or unpaired t-test. For comparisons of categorical variables, frequency tables and chi-square tests were used.

\section{Results:-}

100 patients were enrolled into the study (39 patients with MM, 30 patients with NHL, 10 patients with HD and 21 patients with ALL) (table 1). There were 50 males and 50 females, 55 patients aged <45 years, 21 aged 45-55 years and 24 aged $>55$ years (table 5). Healthy control group included participants without known cardiovascular disease, hypertension or DM. They were matched 1:1 based on age, sex, blood pressure and surface area with the cases. Positive control included 50 patients with lymphoproliferative disorders who received chemotherapy without undergoing BMT. They included 25 males and 25 females, 46\% had MM, 42\% had NHL and 12\% had HD. 72 patients underwent autologus and 21 patient underwent allogeneic stem cell transplantation (figure 1).

Table 1:- Percentages of patients with lymphoproliferative disorders in our study:

\begin{tabular}{|c|c|c|}
\hline \multicolumn{2}{|c|}{ Lymphoproliferative disorder } & \% \\
\hline MM & 39 & 39.00 \\
\hline NHL & 30 & 30.00 \\
\hline HD & 10 & 10.00 \\
\hline TLL & 21 & 21.00 \\
\hline
\end{tabular}




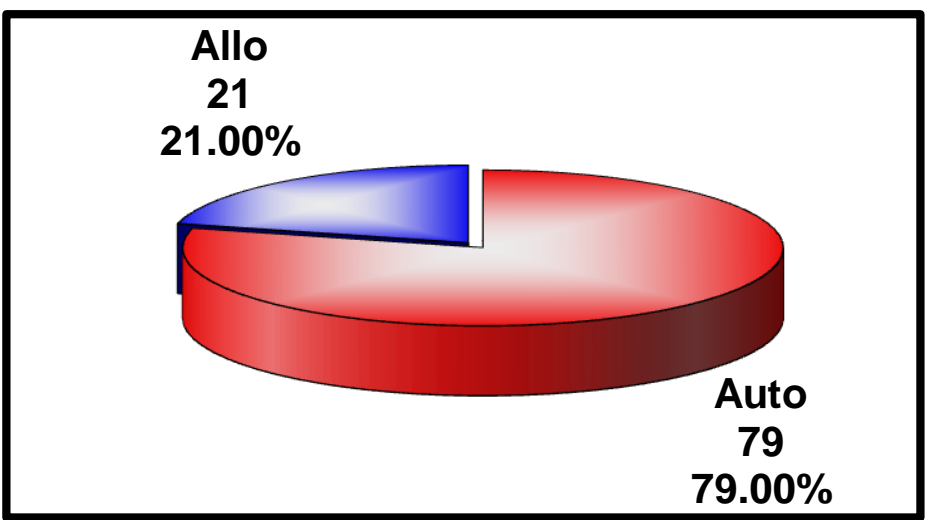

Figure 1:- Percentages of autologus and allogeneic stem cell transplant patients in our study group.

The mean ejection fraction of cases after transplant is significantly reduced compared to the negative control group with highly statistically significant difference $(\mathrm{P}$-value $<0.001)$ (table 2$)$. There is a highly statistically significant difference between ejection fraction in the initial echocardiography before therapy in all patients and the follow up echocardiography after either chemotherapy only in group III or transplanted patients in group I (P-value<0.001).

Also, these results show that group I (transplant group) had higher initial ejection fractions compared to group III with highly statistically significant difference by using unpaired t-test (P-value <0.001) (table 3).

Table 2:- The mean ejection fraction of group I patients that underwent transplant and that of healthy group II (negative control) included in the study:

\begin{tabular}{|l|c|c|c|c|}
\hline \multirow{2}{*}{ ECHO } & \multicolumn{2}{|c|}{ GROUPS } & \multicolumn{2}{c|}{ T-test } \\
\cline { 2 - 5 } & $\begin{array}{c}\text { Group I (cases) after } \\
\text { transplant }\end{array}$ & $\begin{array}{c}\text { Group II } \\
\text { (Negative control) }\end{array}$ & T & P-value \\
\hline Range & $49-70$ & $59-75$ & -5.322 & $<0.001^{*}$ \\
\hline Mean \pm SD & $59.590 \pm 6.299$ & $66.760 \pm 4.728$ & & \\
\hline
\end{tabular}

Table 3:- Correlation between the ejection fraction in the initial echocardiography before therapy in all patients and its decline in the follow up echo after either chemotherapy only in group (3) or transplant in group (1):

\begin{tabular}{|c|c|c|c|c|c|}
\hline \multirow{2}{*}{\multicolumn{2}{|c|}{ Echo }} & \multirow{3}{*}{$\begin{array}{l}\begin{array}{c}\text { Group I } \\
\text { (cases) }\end{array} \\
55-75\end{array}$} & \multirow{3}{*}{$\begin{array}{c}\begin{array}{c}\text { Group III } \\
\text { (Positive control) }\end{array} \\
52-73\end{array}$} & \multicolumn{2}{|c|}{ T-test } \\
\hline & & & & $\mathrm{T}$ & $\mathrm{P}$-value \\
\hline Initial echo & Range & & & \multirow[t]{2}{*}{5.261} & \multirow[t]{2}{*}{$<0.001^{*}$} \\
\hline & Mean \pm SD & $67.290 \pm 4.123$ & $62.880 \pm 6.033$ & & \\
\hline Follow up echo & Range & $49-70$ & $45-62$ & \multirow[t]{2}{*}{5.883} & \multirow[t]{2}{*}{$<0.001^{*}$} \\
\hline & Mean \pm SD & $59.590 \pm 6.299$ & $53.820 \pm 4.084$ & & \\
\hline Paired difference & Mean \pm SD & $7.700 \pm 5.519$ & $9.060 \pm 5.464$ & & \\
\hline \multirow[t]{2}{*}{ Paired samples test } & $\mathrm{T}$ & 13.953 & 11.725 & & \\
\hline & P-value & $<0.001^{*}$ & $<0.001^{*}$ & & \\
\hline
\end{tabular}

Conditioning regimens used during transplant included high dose melphalan in 39 patients with multiple myeloma, Cyclophosphamide/Carboplatin/Etoposide in 36 patients with NHL and HD, Fludarabine/Busulfan and TBI-based regimens in patients with ALL (table 4).

Table 4:- Percentages of conditioning regimens used in our study:

\begin{tabular}{|c|c|c|}
\hline \multicolumn{3}{|c|}{ Conditioning regimen } \\
\hline & $\mathbf{N}$ & $\%$ \\
\hline High dose melphalan & 39 & 39.00 \\
\hline Cyclophosphamide/Carboplatin/Etoposide & 36 & 36.00 \\
\hline Fludarabine/Busulfan & 14 & 14.00 \\
\hline TBI-based & 11 & 11.00 \\
\hline Total & 100 & 100.00 \\
\hline
\end{tabular}


Chemotherapy regimens used prior to transplant included hyperCVAD/Holezer protocols for patients with ALL, VAD/CVAMP for MM, ABVD/GDP for HD and CHOP/DHAP for NHL (figure 2).

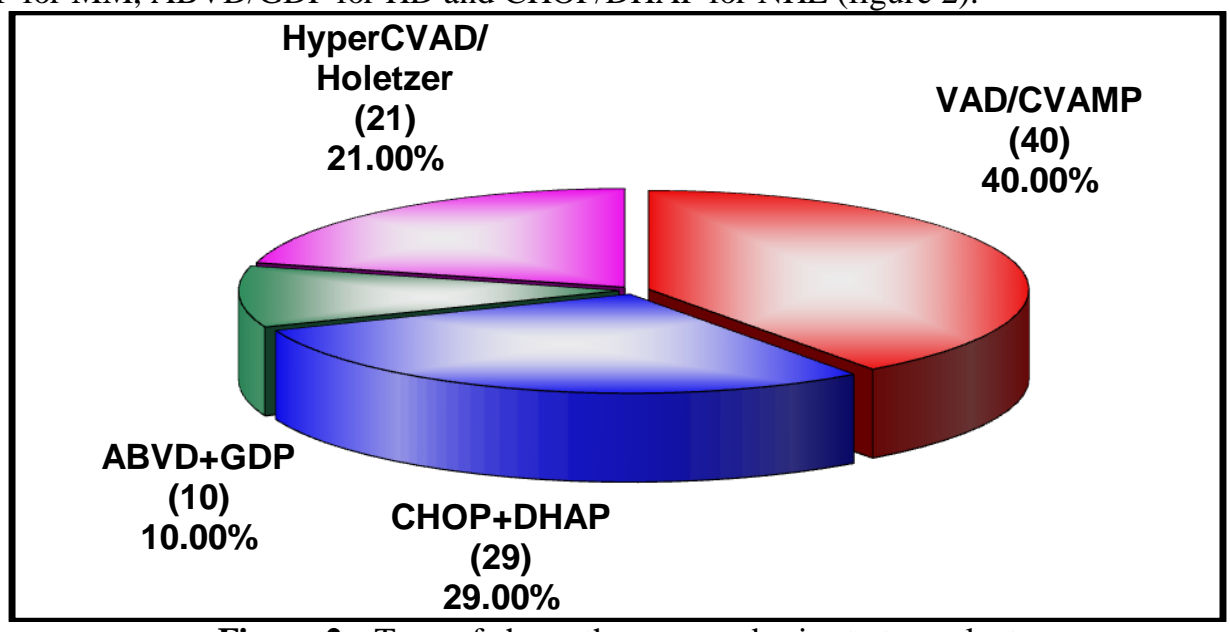

Figure 2:- Type of chemotherapy used prior to transplant

The ejection fraction decline was highly pronounced in older age groups compared to younger group with highly statistically significant difference (P-value 0.001) (table 5). The ejection fraction decline was seen more in male cases compared to females (P-value 0.009) (table 5). Also, the decline of ejection fraction was more in patients with surface area $>1.8$ than those with surface area $<1.8$ (P-value 0.040) (table 5). There is no statistically significant difference between the type of transplant and the decline in ejection fraction (table 5).

Table 5:- Relation of the different age groups, sex, surface area and type of transplant of the cases and the decline in their ejection fraction:

\begin{tabular}{|c|c|c|c|c|c|c|c|c|}
\hline & \multicolumn{6}{|c|}{ EF decline } & \multirow{2}{*}{\multicolumn{2}{|c|}{ Chi-Square }} \\
\hline & \multicolumn{2}{|c|}{ Negative } & \multicolumn{2}{|c|}{ Positive } & \multicolumn{2}{|c|}{ Total } & & \\
\hline & $\mathbf{N}$ & $\%$ & $\mathbf{N}$ & $\%$ & $\mathbf{N}$ & $\%$ & $\mathbf{X}^{2}$ & P-value \\
\hline Age Groups & & & & & & & 13.638 & $0.001 *$ \\
\hline$<45$ Years & 52 & 63.41 & 3 & 16.67 & 55 & 55.00 & & \\
\hline 45-55 Years & 15 & 18.29 & 6 & 33.33 & 21 & 21.00 & & \\
\hline$>55$ Years & 15 & 18.29 & 9 & 50.00 & 24 & 24.00 & & \\
\hline Total & 82 & 100.00 & 18 & 100.00 & 100 & 100.00 & & \\
\hline Sex & & & & & & & 6.775 & $0.009^{*}$ \\
\hline Male & 36 & 43.90 & 14 & 77.78 & 50 & 50.00 & & \\
\hline Female & 46 & 56.10 & 4 & 22.22 & 50 & 50.00 & & \\
\hline Total & 82 & 100.00 & 18 & 100.00 & 100 & 100.00 & & \\
\hline Surface area $>1.8$ & & & & & & & 4.225 & $0.040^{*}$ \\
\hline Negative & 40 & 48.78 & 4 & 22.22 & 44 & 44.00 & & \\
\hline Positive & 42 & 51.22 & 14 & 77.78 & 56 & 56.00 & & \\
\hline Total & 82 & 100.00 & 18 & 100.00 & 100 & 100.00 & & \\
\hline Type of transplant & & & & & & & 2.013 & 0.156 \\
\hline Auto & 67 & 81.71 & 12 & 66.67 & 79 & 79.00 & & \\
\hline Allo & 15 & 18.29 & 6 & 33.33 & 21 & 21.00 & & \\
\hline Total & 82 & 100.00 & 18 & 100.00 & 100 & 100.00 & & \\
\hline
\end{tabular}

There is a statistical significance between the decline in ejection fraction and the conditioning regimen used for the transplant with patients in the TBI-based regimens are more affected followed by patients who received high dose cyclophosphamide (table 6).

Comparing TBI-based regimens to non-TBI regimens there is highly statistically significant difference between the decline in ejection fraction and the use of TBI (P-value 0.012) (table 7). 
Table 6:- Correlation between the conditioning regimens used to prepare group (1) patients and the decline in ejection fractions:

\begin{tabular}{|c|c|c|c|c|c|c|c|c|}
\hline \multirow[t]{3}{*}{ Conditioning regimen } & \multicolumn{6}{|c|}{ EF decline } & \multirow{2}{*}{\multicolumn{2}{|c|}{ Chi-Square }} \\
\hline & \multicolumn{2}{|c|}{ Negative } & \multicolumn{2}{|c|}{ Positive } & \multicolumn{2}{|c|}{ Total } & & \\
\hline & $\mathbf{N}$ & $\%$ & $\mathbf{N}$ & $\%$ & $\mathbf{N}$ & $\%$ & $\mathbf{X}^{2}$ & P-value \\
\hline Melphalan & 36 & 43.90 & 3 & 16.67 & 39 & 39.00 & 8.587 & $0.035^{*}$ \\
\hline Endoxan/Carboplatin/Etoposide & 29 & 35.37 & 7 & 38.89 & 36 & 36.00 & & \\
\hline Fludarabine/Busulfan & 11 & 13.41 & 3 & 16.67 & 14 & 14.00 & & \\
\hline TBI-based & 6 & 7.32 & 5 & 27.78 & 11 & 11.00 & & \\
\hline Total & 82 & 100.00 & 18 & 100.00 & 100 & 100.00 & & \\
\hline
\end{tabular}

Table 7:- Correlation between the use of TBI-based conditioning regimens to prepare cases and decline in their ejection fraction:

\begin{tabular}{|c|c|c|c|c|c|c|c|c|}
\hline \multirow[t]{3}{*}{ Conditioning regimen TBI } & \multicolumn{6}{|c|}{ EF decline } & \multirow{2}{*}{\multicolumn{2}{|c|}{ Chi-Square }} \\
\hline & \multicolumn{2}{|c|}{ Negative } & \multicolumn{2}{|c|}{ Positive } & \multicolumn{2}{|c|}{ Total } & & \\
\hline & $\mathbf{N}$ & $\%$ & $\mathbf{N}$ & $\%$ & $\mathbf{N}$ & $\%$ & $\mathbf{X}^{2}$ & P-value \\
\hline Negative & 76 & 92.68 & 13 & 72.22 & 89 & 89.00 & 6.312 & $0.012 *$ \\
\hline Positive & 6 & 7.32 & 5 & 27.78 & 11 & 11.00 & & \\
\hline Total & 82 & 100.00 & 18 & 100.00 & 100 & 100.00 & & \\
\hline
\end{tabular}

\section{Discussion:-}

The current standard for cardiac monitoring during cancer therapy is mainly based on LVEF assessment, but it can underestimate early potentially progressive cardiotoxicity, and preserved cardiac function is generally required for enrollment in clinical trials of high dose chemotherapy. This is commonly defined as LVEF $>50 \%$ and no other significant cardiac disease.

Many studies reported a significant association between pretransplant LVEF and cardiotoxicity. However, measurement of LVEF before high dose chemotherapy is not the only determinant factor as $2 / 3$ major cardiac events reported in patients with normal $\mathrm{LVEF}^{2}$.

In the current study, we found that the mean ejection fraction of cases after transplant is significantly reduced compared to the negative control group with highly statistically significant difference ( $\mathrm{P}$-value $<0.001)$. Also, there is a highly statistically significant difference between ejection fraction in the initial echocardiography before therapy in all patients and the follow up echocardiography after either chemotherapy only in group III or transplanted patients in group I (P-value<0.001).

A wide variety of conditioning regimens have been used prior to stem cell transplantation. Including, TBI-based regimens and high-dose cyclophosphamide, and cardiac toxicity has been associated mostly with the use of these regimens with a wide spectrum of incidence, manifestation and severity.

HD cyclophosphamide-associated cardiac toxicity is thought to depend upon toxic endothelial damage followed by extravasation of toxic metabolites with resultant myocyte damage and interstitial hemorrhage and edema. HD cyclophosphamide-associated cardiotoxicity occurs during or soon after (within 3 weeks) administration. It is manifested clinically as acute or subacute onset of congestive heart failure (CHF) with pulmonary congestion, weight gain and oliguria. Pericardial effusion, in some cases with cardiac tamponade. Although HD cyclophosphamide-associated cardiac toxicity is potentially reversible, in patients who develop severe, progressive $\mathrm{CHF}$, this complication may lead to death within few weeks ${ }^{2}$.

In our study, we found that ejection fraction decline is higher in patients with a body surface area $>1.8$ than those with a body surface area $<1.8$ reflecting a higher dosage of chemotherapeutic agents used in those patients.

In fact, optimal chemotherapy dosing in obese patients is one of the most controversial aspects of HD chemotherapy since obese patients have altered pharmacokinetics for many medications including chemotherapeutic agents when compared with the non-obese. 
Indeed, association of obesity with cardiac toxicity can be spotted throughout the literature. As a practical approach, reduction of HD cyclophosphamide dosage in patients whose actual BW exceeds by $>20 \%$ of the IBW is a safety measure that doesn't affect the efficacy of chemotherapy and minimizes the risk of toxicity ${ }^{2}$.

In the current study, the ejection fraction decline was highly pronounced in older age groups compared to younger group with highly statistically significant difference (P-value 0.001 ). The ejection fraction decline was seen more in male cases compared to females (P-value 0.009).

The comparison between autologus and allogeneic recipients is especially important. More pronounced cardiotoxic effects could be expected in allogeneic recipients as a result of a different spectrum of conditioning regimens, infectious complications and also an effect of GVHD.

In our study, no difference was found between the incidence of EF decline and the type of transplant. However, the number of allo HSCT in this study is much fewer than the auto patients.

Hernstein et al found that despite the speculation of higher incidence of cardiotoxic complications in patients undergoing allo-HSCT, a higher incidence heart failure was found in autologus recipients in their study. However, the number of autologus patients in their study was small ${ }^{4}$.

There is a statistical significance between the decline in ejection fraction and the conditioning regimen used for the transplant with patients in the TBI-based regimens are more affected followed by patients who received high dose cyclophosphamide.

Comparing TBI-based regimens to non-TBI regimens there is highly statistically significant difference between the decline in ejection fraction and the use of TBI (P-value 0.012).

The epidemiology of RICVD is complicated by the continual improvements in radiation dosimetry and shielding that tend to reduce cardiovascular exposure and the latent effects of radiation, which takes years or decades to manifest.

Several large studies have been published over the last few years that analyzed the outcome of RT administered between one and four decades ago.

Radiation-induced pericarditis is the earliest form of RICVD to occur following mediastinal irradiation. It may occur in either of two forms, early and acute or delayed and chronic, which should be regarded from a histopathological standpoint as two distinct disease entities 5 .

The risk of radiation-induced cardiomyopathy increases after 5 years, but it can evolve decades after initial $\mathrm{RT}^{6}$. Pathologically, RICM is characterized by inflammation followed by the development of a diffuse, patchy interstitial fibrosis of the myocardium and effacement of perimyocyte and endothelium ${ }^{7}$.

More recently, researchers at the Netherlands Cancer Institute found a stepwise decrease in 30-year cumulative incidence of valvular heart disease (VHD) corresponding to diminishing doses of RT, from $12.4 \%$ at doses greater than $40 \mathrm{~Gy}$ to $3.0 \%$ at doses less than $30 \mathrm{~Gy}^{8}$.

A large case control study of breast cancer survivors in Denmark and Sweden undertaken in 2013 found that the risk of a major $\mathrm{CHD}$ event begins to increase within the first 5 years post-treatment and continues to significantly exceed that of the general population through at least 20 years of follow-up. These patients experienced increased risk of angina pectoris, MI, and sudden cardiac death despite having been treated with a modest mean heart dose of 3.6 Gy RT between 1958 and 2001 .

Another large study of women in Denmark and Sweden ( $n=35000)$ comparing incidences of MI in breast cancer survivors observed an incidence ratio of 1.22 in patients undergoing left-sided $v s$ right-sided $\mathrm{RT}^{10}$. 
In the current study $36.4 \%$ of lymphoma patients that underwent autologus transplant with cyclophosphamide-based regimens developed a decline in their LVEF. Previous reports showed that CY cardiotoxicity is correlated with the dose of CY is being higher in patients received $200>120>100 \mathrm{mg} / \mathrm{kg}^{11}$.

In a previous report, higher age, administration of cyclophosphamide and higher glucose concentrations represented independent risk factors for the worsening of left ventricular diastolic function ${ }^{12}$.

Several methods were investigated to reduce anthracycline cardiotoxicity, most cardioprotective strategies are not in common clinical practice. Other approaches to mitigate the cardiotoxic impact of anthracyclines employ potentially cardioprotective medications, such as ACE inhibitors and beta blockers. Although promising data have been published recently, convincing evidence from large randomized and prospective trials is still needed ${ }^{3}$

There are some limitations of our current analysis. First, this was a retrospective study from a single institution. Second, early CY-induced cardiotoxicity appears to be characterized by LV diastolic rather than systolic dysfunction.

\section{Conclusion:-}

LVEF was reduced in patients with lymphoproliferative disorders after autologus and allogeneic hematopoietic stem cell transplantation and was correlated with the use of TBI-based conditioning regimens and high-dose cyclophosphamide.

\section{References:-}

1. Lehmann S, Isberg B, Ljungman P, et al. Cardiac systolic function before and after hematopoietic stem cell transplantation. Bone marrow transplantation 2000; 26(2): 187-192.

2. Morandi P, Ruffini PA, Benvenuto GM. Cardiac toxicity of high-dose chemotherapy. Bone marrow transplantation 2005; 35(4): 323-324.

3. Roziakova L, Mistrik M, Batorova A. Can We Predict Clinical Cardiotoxicity with Cardiac Biomarkers in Patients After Haematopoietic Stem Cell Transplantation? Cardiovasc Toxicol 2015; 15:210-216.

4. Hertenstein B, Stefanic M, Schmeiser T et al. Cardiac toxicity of bone marrow transplantation: predictive value of cardiologic evaluation before transplant. J Clin Oncol 1994; 12:998-1004.

5. Cuomo JR, Sharma GK, Conger PD et al. Novel concepts in radiation-induced cardiovascular disease. World journal of cardiology 2016; 26; 8(9): 504-519.

6. Aleman BM, van den Belt-Dusebout AW, De Bruin ML et al. Late cardiotoxicity after treatment for Hodgkin lymphoma. Blood 2007;109: 1878-1886.

7. Heidenreich PA, Kapoor JR. Radiation induced heart disease: systemic disorders in heart disease. Heart 2009; 95: 252-258.

8. Cutter DJ, Schaapveld M, Darby SC et al. Risk of valvular heart disease after treatment for Hodgkin lymphoma. J Natl Cancer Inst 2015; 107(4):djv008.

9. Darby SC, Ewertz M, McGale $\mathbf{P}$ et al. Risk of ischemic heart disease in women after radiotherapy for breast cancer. NEngl J Med2013; 368: 987-998.

10. McGale P, Darby SC, Hall P et al. Incidence of heart disease in 35,000 women treated with radiotherapy for breast cancer in Denmark and Sweden. Radiother Oncol 2011; 100: 167-175.

11. Ishida S, Doki N, Shingai $\mathbf{N}$ et al. The clinical features of fatal cyclophosphamide-induced cardiotoxicity in a conditioning regimen for allogeneic hematopoietic stem cell transplantation (allo-HSCT). Ann Hematol 2016; 95:1145-1150.

12. Poręba M, Gać P, Usnarska-Zubkiewicz L. Echocardiographic evaluation of the early cardiotoxic effect of hematopoietic stem cell transplantation in patients with hematologic malignancies. Leukemia and lymphoma 2016; 57(9): 2119-2125. 